\title{
Pulsating hemorrhagic varicose veins caused by tricuspid valve regurgitation: report of a case treated by laser ablation and foam sclerotherapy
}

\author{
Atsushi Guntani $^{*} \mathbb{D}$, Sho Yamashita and Shinsuke Mii
}

\begin{abstract}
Background: Varicose veins are one of the most common disease; however, secondary varicose veins caused by tricuspid valve regurgitation (TVR) are rare.

Case presentation: A patient who developed pulsating bleeding from superficial varicose veins due to TVR was successfully treated by endovenous laser ablation (EVLA) of the great saphenous vein (GSV) and repeated foam sclerotherapy of varicose veins. There were no complications, such as rebleeding or recanalization of the GSV during the 1-year follow-up period.

Conclusions: We herein report a rare case of pulsatile hemorrhagic varicose veins caused by TVR that was successfully managed by combined treatment of EVLA and foam sclerotherapy. When pulsatile varicose veins are found, the presence of TVR should be suspected.
\end{abstract}

Keywords: Endovenous laser ablation, Varicose vein, Tricuspid valve regurgitation

\section{Background}

Most varicose veins are caused by primary valvular insufficiency. It is reported that only about $5-20 \%$ of varicose veins occur secondary to conditions, such as deep vein thrombosis, pelvic tumor or arteriovenous fistula [1,2]. In particular, secondary varicose veins caused by tricuspid valve regurgitation (TVR) are extremely rare [3-5]. We successfully treated a patient with pulsatile hemorrhagic varicose veins caused by TVR using endovenous laser ablation (EVLA) of the great saphenous vein (GSV) and repeated foam sclerotherapy of varicose veins.

*Correspondence: aguntani@yahoo.co.jp

Department of Vascular Surgery, Saiseikai Yahata General Hospital, 5-9-27

Haruno-machi, Yahatahigashi-ku, Kitakyushu 805-8527, Japan

\section{Case presentation}

A 69-year-old male patient was admitted for pulsating bleeding from superficial varicose veins of the left dorsal foot. After compression hemostasis, ultrasonography revealed pulsatile regurgitation in the left GSV (Fig. 1). He had a history of ligation of two incompetent perforating veins in the left thigh, and high ligation and stripping of the right GSV. In a previous clinical chart, pulsatile palpation of the right GSV with significant postoperative hematoma was described in the surgical record; however, no further examination was performed at that time.

Dilated superficial varicose veins and pigmentation were observed from the left lower leg to the dorsal foot, equivalent to $\mathrm{C}_{4 \mathrm{a}}$, as assessed using the clinical, etiological, anatomical and pathological (CEAP) classification. Ultrasonography revealed regurgitation in the left deep veins, from the common femoral to the popliteal, and perforators in the left lower leg, in addition to the left 


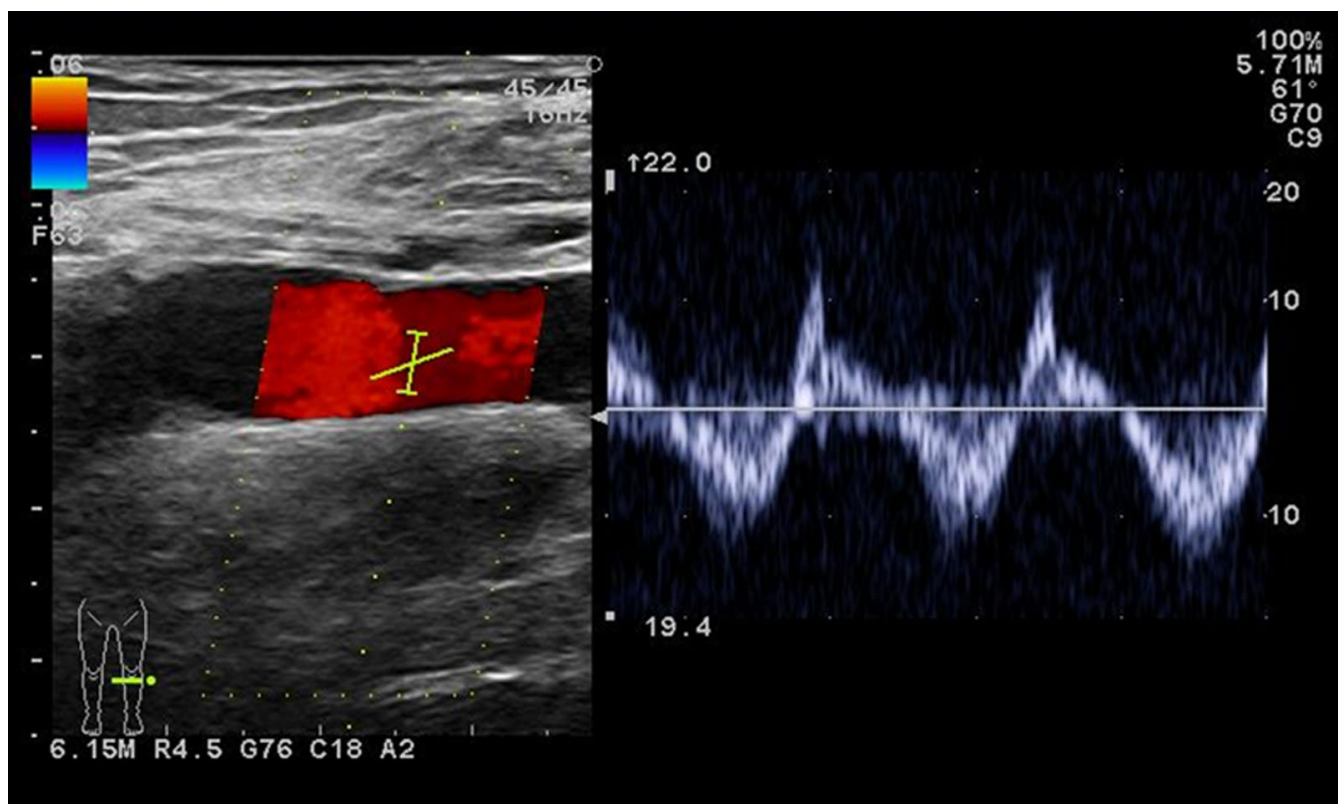

Fig. 1 Ultrasonography showed pulsatile regurgitation in the left GSV

GSV. We clinically suspected arteriovenous fistula and the patient was admitted for a detailed hemodynamic examination. Computed tomography revealed the presence of congestive liver and inferior vena cava dilatation; however, no arteriovenous fistula was observed (Fig. 2A, B). Subsequent echocardiography showed prominent enlargement of the right atrium and right ventricle, and severe TVR (Fig. $2 \mathrm{C}$ ). Thus, the pulsatile venous blood flow was considered to be derived from TVR.

EVLA was performed on the left GSV of the thigh using a $1470 \mathrm{~nm}$ diode laser with a radial 2-ring fiber (ELVeS Radial 2ring ${ }^{\mathrm{TM}}$ fiber, CeramOptec GmbH, Germany) under general anesthesia with tumescent local anesthesia $(430 \mathrm{~mL}$ saline $+50 \mathrm{~mL}$ lidocaine $1 \%+20 \mathrm{~mL}$ sodium hydrotricarbonate 7\%). Subsequently, three incompetent perforating veins in the left lower leg were identified under ultrasound guidance, and then ligated and dissected. The puncture site of the introducer sheath and the incision in the lower leg bled easily; however, hemostasis could be obtained by manual compression. After the operation, the patient rested on the bed with the left lower limb compressed by an elastic bandage. Duplex sonography confirmed the success of EVLA of the left GSV and the patency of the deep vein system (Fig. 3). Two days after EVLA, foam sclerotherapy using $1 \%$ polidocanol (dilution ratio for sclerosant to air: 1:3) was performed for the superficial varicose veins of the left foot, including the bleb, where bleeding was encountered at the time of admission.
The patient used a compression stocking and was followed regularly as an outpatient. It was necessary to perform foamed sclerotherapy three times for other superficial varicose veins of the left lower limb; however, the examination using duplex ultrasonography had not detected recanalization of the occluded left GSV and bleeding from the varicose veins was not observed during the 1-year follow-up period.

\section{Discussion}

Tricuspid insufficiency results in elevated central venous pressure, and various clinical signs, such as enlarged liver, peripheral edema, and ascites are observed. According to past case reports, in cases of tricuspid insufficiency, pulsatile veins have been observed in the neck, forearm, and forehead [6, 7]. In our case, computed tomography revealed congestive liver, pleural effusion, and ascites; however, there were no obvious subjective symptoms other than pulsating bleeding from the superficial varicose veins of the left foot.

Our patient had a history of high ligation and stripping of the right GSV. The right GSV was pulsating according to the surgical record. Significant hematoma occurred as a complication after the operation. It seems that TVR was already present; however, no further examination was performed. Right ventricular cardiomyopathy caused by arrhythmia was the main pathology of the disease and the main cause of right ventricular enlargement and decreased right ventricular contractility, which led to TVR. Therefore, it was highly possible that even if 

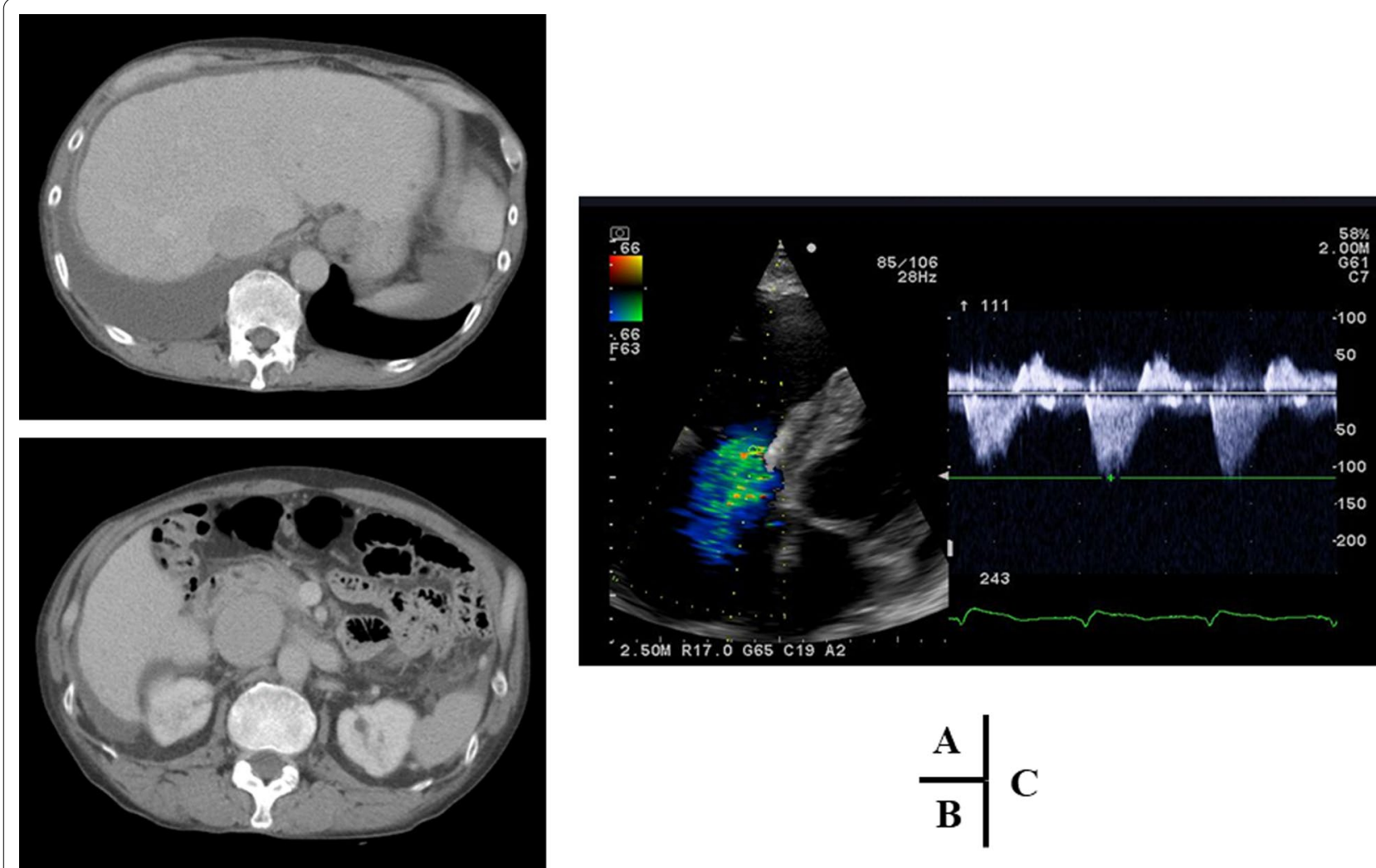

Fig. 2 Computed tomography revealed the presence of congestive liver and inferior vena cava dilatation (A, B). Echocardiography showed prominent enlargement of the right atrium and right ventricle, and severe TVR (C)

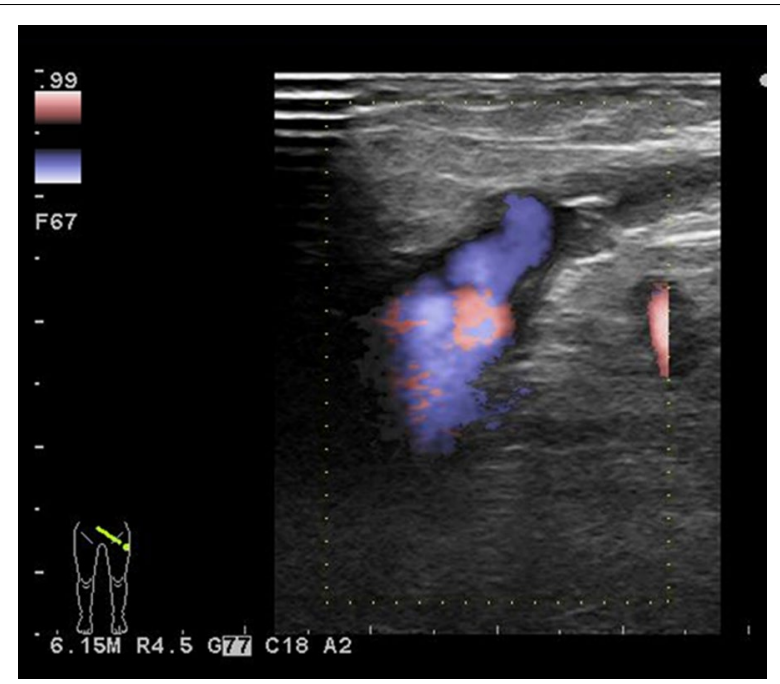

Fig. 3 Follow-up duplex sonography revealed that the success of EVLA of the left GSV and the patency of the deep vein system

tricuspid valve replacement surgery had been performed, it would not have contributed to the reduction in the venous pressure. Drug therapy with diuretics (torsemide,
$8 \mathrm{mg}$, daily) and cardiac stimulants (pimobendane, $5 \mathrm{mg}$, daily) was introduced after the bleeding event involving the varicose veins.

In previous studies, some cases of pulsatile varicose veins secondary to TVR were treated by approaches, such as EVLA alone and saphenofemoral ligation with foam sclerotherapy; however, other cases were treated conservatively by compression therapy with elastic stockings, in consideration of the bleeding due to venous hypertension and cardiac risk during the operation [1, 8-11]. Our case has a history of both stripping and EVLA of the GSV; however, EVLA seems to be associated with a lower risk of bleeding, considering the complication of hematoma after stripping of the GSV. In addition, since the course of this case was favorable during the 1-year follow-up period, it was considered that EVLA showed satisfactory venous sealing efficiency, even in the presence of high venous pressure.

Deep vein regurgitation was also observed in our case, so even after EVLA of the GSV, regurgitation through the incompetent perforating vein and rebleeding from varicose veins may occur. Therefore, it was considered that ligation of the incompetent perforating veins and foam sclerotherapy for the superficial varicose veins were 
effective. Since there is not enough evidence concerning the long-term outcomes of EVLA for varicose veins caused by TVR, further follow-up will be necessary.

\section{Conclusion}

We herein report a rare case of pulsatile hemorrhagic varicose veins caused by TVR that was successfully treated by EVLA and foam sclerotherapy. When pulsatile varicose veins are found, the presence of TVR should be suspected.

\section{Abbreviations}

TVR: Tricuspid valve regurgitation; GSV: Great saphenous vein; EVLA: Endovenous laser ablation; CEAP: Clinical, etiological, anatomical and pathological.

\section{Acknowledgements}

Not applicable.

\section{Authors' contributions}

AG contributed to writing the manuscript. SM supervised the study. All authors collected the data, discussed the content of the manuscript. All authors read and approved the final manuscript.

\section{Funding}

This study was not funded.

\section{Availability of data and materials}

All data sets supporting the conclusions of this article are included in this published article.

\section{Declarations}

Ethics approval and consent to participate

This study was carried out in accordance with the principles of the Declaration of Helsinki.

\section{Consent for publication}

Informed consent was obtained from the patient for publication of this article.

\section{Competing interests}

The authors declare that they have no competing interests.
Received: 2 July 2021 Accepted: 31 August 2021

Published online: 03 September 2021

\section{References}

1. Swoboda S, Schumann $H$, Kiritsi D. A leg ulcer with pulsating varicose veins - from the legs to the heart. Int Wound J. 2018;15:62-64.1.

2. Bhatti AM, Siddique K, Bashir RA, Sajid MT, Mustafa Q, Hussain SM, Shukr I, Ahmed M. Unusual causes of secondary varicose veins. J Ayub Med Coll Abbottabad. 2013;25(3-4):81-85.3.

3. Fred $\mathrm{HL}$. The tricuspid insufficiency-pulsating varicocele connection: a syndrome and its history. Texas Heart Inst J. 2017:44:302-305.4.

4. Barnett N, Collyer TC, Weston M, Spark J. Pulsatile varicose veins. J R SoC Med. 2000;93:29-30.5.

5. Dayantas J, Liatas AC, Lazarides M. Pulsatile varicose veins caused by tricuspid valve regurgitation. Phlebology. 1990;5(6):189-191.6.

6. Brickner PW, Scudder WT, Weinrib M. Pulsating varicose veins in functional tricuspid insufficiency: case report and venous pressure tracing. Circulation. 1962;115:126-129.7.

7. Abu-Yousef MM, Kakish ME, Mufid M. Pulsatile venous doppler flow in lower limbs: highly indicative of elavated right atrium pressure. AJR. 1996;167:977-980.8.

8. Li X, Feng Y, Liu Y, Zhang F. Varicose veins of the lower extremity secondary to tricuspid regurgitation. Ann Vasc Surg. 2019;60:477.e1-477.e6. https://doi.org/10.1016/j.avsg.2019.02.052 (Epub 2019 May 7. 9).

9. Chihara S, Sawada K, Tomoeda H, Aoyagi S. Pulsatile varicose veins secondary to severe tricuspid regurgitation: report of a case successfully managed by endovenous laser treatment. Ann Vasc Surg. 2017;39:286. e14. https://doi.org/10.1016/j.avsg.2016.06.026 (Epub 2016 Sep 22.10).

10. Lawaetz M, Serup J, Lawaetz B, Bjoern L, Blemings A, Eklof B, Rasmussen L. Comparison of endovenous ablation techniques, foam sclerotherapy and surgical stripping for great saphenous varicose veins. Extended 5-year follow-up of a RCT. Int Angiol. 2017;36(3):281-288.11.

11. Casian D, Gutsu E, Culiuc V. Surgical treatment of severe chronic venous insufficiency caused by pulsatile varicose veins in a patient with tricuspid regurgitation. Phlebology. 2009;24(2):79-81.12.

\section{Publisher's Note}

Springer Nature remains neutral with regard to jurisdictional claims in published maps and institutional affiliations.

\section{Submit your manuscript to a SpringerOpen ${ }^{\circ}$ journal and benefit from:}

- Convenient online submission

- Rigorous peer review

- Open access: articles freely available online

- High visibility within the field

- Retaining the copyright to your article

Submit your next manuscript at $\boldsymbol{\nabla}$ springeropen.com 